\title{
Young homeless women encountered physical and individual barriers in obtaining health care
}

Ensign J, Panke A. Barriers and bridges to care: voices of homeless female adolescent youth in Seattle, Washington, USA. J Adv Nurs 2002 Jan;37:166-72.

\section{QUESTION: What are the common sources of advice, health seeking behaviours, and self perceived barriers to health care of young homeless women?}

Source of funding: not stated.

For correspondence:

Dr J Ensign, University

of Washington, Seattle,

WA, USA. bjensign@u.washington.edu rant of personal healthcare issues. Communication issues and they were reprimanded when they looked through their charts. The women preferred the providers to be information confidential. They felt providers were often "too pushy" and treated homeless women as being igno-

\section{Design}

Ethnography.

\section{Setting}

Youth clinic within a community health clinic in Seattle, Washington, USA.

\section{Participants}

20 women between 14 and 23 years of age (mean age 18 y) who were homeless or had been homeless in the past 12 months.

\section{Methods}

Each woman had a semistructured interview with 1 of 2 research nurses about sources of advice, health seeking behaviours, and access to care issues. Each interview concluded with the nurse addressing the health concerns of the woman. Interviews were tape recorded and preliminarily analysed. 6 women were recruited from the larger group to participate in 3 focus groups to explore themes and topics identified during the interviews in more depth. Focus groups were also tape recorded. Women received US $\$ 5$ for participating in the semistructured interviews and $\$ 10$ for the focus groups.

\section{Main findings}

Sources of advice: most women sought advice about a health problem or concern from a female friend or relative, particularly their mothers. Some women reported consulting a medical reference book in a library or bookstore or calling an Ask-a-Nurse phone consulting service. All women in the focus group agreed they preferred seeking advice from women rather than men. Health seeking behaviours: most women tried to manage their ailments with self care and went to a clinic as a last resort. The decision to go to a clinic was made when they could no longer continue their life in their normal manner. They noted that men were more hesitant to attend clinics. Access to care issues: women stated that if they attended regular clinics not specifically for homeless youth, they encountered structural barriers in the form of questions about consent for care, permanent addresses, personal identification, and health insurance. Several women stated that being physically sick was an individual barrier to seeking health care, and that having a friend accompany them was important. The women felt the overall characteristics of clinics were impersonal or hostile to them. They knew if their privacy was not respected by the way clinic staff interacted with them in the waiting

with providers included use of medical terminology, provision of conflicting advice or diagnoses, and incongruency between the biomedical understanding of disease by the provider and the womens' belief systems.

\section{Conclusions}

Young homeless women described structural and personal barriers and facilitators to obtaining health care. They emphasised needing the accompaniment of friends, designated clinics for homeless youths, female providers, greater respect, a non-judgmental and confidential approach, and better listening from providers.

\section{COMMENTARY}

The qualitative study by Ensign and Panke provides important information about barriers and facilitators for homeless adolescent women in accessing health care, as well as their health seeking behaviours. The findings are consistent with those of other studies on homeless people and people living in poverty, and the literature on care seeking and help seeking behaviours and processes. Homeless people are often perceived as considering health care to be a low priority compared with such higher priority daily needs as finding food and shelter. ${ }^{1}$ The results of this study provide evidence that self care and health information seeking are naturally used first by homeless young women before accessing formal care. This "natural delay" in seeking health care has been associated in the literature with a person's way of making sense of their illness. ${ }^{2}$

An ethnographic approach is particularly important to enhance our understanding of the health beliefs of homeless people and to reveal the culturally embedded norms that guide their health seeking actions. Drawing on the best practice concept of cultural competence, the authors framed the findings for application by clinicians, most notably those findings related to provider power within client-provider interactions. These findings also support the need for further identification of ways in which the social support network of homeless people contributes to health care access by the homeless.

This study provides important direction for clinical practice. Healthcare providers must recognise homeless adolescent women as having some expertise in the management of their own illnesses. Such an attitude will enable providers to actively listen to and identify the youths' health goals, needs, and beliefs. Providers also need to recognise the inherent competence of homeless adolescents for self care strategies in relation to their own health.

Betty Da Silva, RN, MSN

Clinical Nurse Specialist - Adult/Older Adult Services Vancouver Coastal Health Authority Vancouver Community Vancouver, British Columbia, Canada trusting, respectful, and non-judgmental, and to keep 